\title{
Transvalencin Z, a New Antimicrobial Compound with Salicylic Acid Residue from Nocardia transvalensis IFM 10065
}

\author{
Akira Mukai, Toshio Fukai, Yuko Matsumoto, Jun Ishikawa, Yasutaka Hoshino, \\ Katsukiyo Yazawa, Ken-ichi Harada, Yuzuru Mikami
}

Received: April 27, 2006 / Accepted: June 9, 2006

(C) Japan Antibiotics Research Association

\begin{abstract}
Transvalencin $\mathrm{Z}$ was isolated from a culture broth of Nocardia transvalensis IFM 10065, a clinical isolate from a Japanese patient with actinomycotic mycetoma. The transvalencin $\mathrm{Z}$ structure was determined using NMR and mass spectrometric analyses. The structure is similar to a partial structure of siderophores such as mycobactins and nocobactins, but the compound has no cytotoxic activity. Transvalencin $\mathrm{Z}$ shows a strong antimicrobial activity against Gram-positive bacteria, but shows no activity against Gram-negative bacteria, fungi and tumor cells.
\end{abstract}

Keywords transvalencin Z, siderophores, Nocardia transvalensis, nocobactin

\section{Introduction}

Most Nocardia species can cause human infections called nocardiosis. The infections that are caused by Nocardia are considered rare, but infectious cases have been increasingly reported, especially with the increase of immunocompromised patients $[1,2]$. During our screening program of bioactive compounds from pathogenic actinomycetes, we found that most of the culture broth of Nocardia clinical isolates produce anti-tumor compounds that are extractable using hydrophobic solvents such as

Y. Mikami (Corresponding author), A. Mukai, Y. Matsumoto, K. Yazawa: Research Center for Pathogenic Fungi and Microbial Toxicoses, Chiba University, 1-8-1 Inohana, Chuo-ku, Chiba, Chiba 260-8673, Japan, E-mail: mikami@faculty.chiba-u.jp

T. Fukai: School of Pharmaceutical Sciences, Toho University, 2-2-1 Miyama, Funabashi, Chiba 274-8510, Japan ethyl ether or $n$-hexane [3,4]. Our studies suggest that most of the active compounds from these pathogenic bacteria belong to siderophore-class antibiotics such as brasilibactin A [4], madurastatin [5] and asterobactin [6]. These siderophores are characterized as having salicylic acid moieties in their structures. We previously reported that $N$. transvalensis IFM 10065 produces a novel zinc-containing antibiotic, transvalencin A $[6,7]$. Our continuous screening of new bioactive metabolites from that bacterium has led to isolation of a novel antimicrobial compound with a salicylic acid moiety in its structure, designated as transvalencin Z (Fig. 1, 1). In this paper, we describe its production, isolation, physicochemical properties, structural characterization and biological activity.

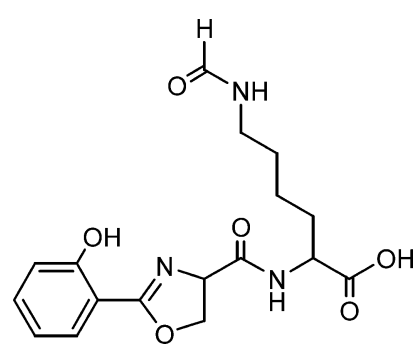

Fig. 1 Structure of transvalencin Z.

J. Ishikawa, Y. Hoshino: Department of Bioactive Molecules, National Institute of Infectious Diseases, 1-23-1 Toyama, Shinjuku-ku, Tokyo 162-8640, Japan

K. Harada: Faculty of Pharmacy, Meijo University, Tempaku, Nagoya 468-8503, Japan 
Table 1 Physicochemical properties of transvalencin Z

\begin{tabular}{ll}
\hline Appearance & White powder \\
Molecular weight & 363 \\
Molecular formula & $\mathrm{C}_{17} \mathrm{H}_{21} \mathrm{~N}_{3} \mathrm{O}_{6}$ \\
HRFAB-MS $(\mathrm{m} / \mathrm{z})$ & \\
Found: & $364.1503(\mathrm{M}+\mathrm{H})^{+}$ \\
Calcd.: & 364.1509 \\
{$[\alpha]_{D}^{25.5}$} & $+15.3^{\circ}(\mathrm{c} 1 \mathrm{mg} / \mathrm{ml}, \mathrm{MeOH})$ \\
UV $\lambda_{\max }^{\mathrm{MeOH}} \mathrm{nm}(\log \varepsilon)$ & $205.5(3.77), 241.0(3.28)$, \\
& $301.5(2.91)$
\end{tabular}

\section{Fermentation and Isolation}

$N$. transvalensis IFM 10065 was cultured as reported previously [6], and the separation of active compounds was monitored by exhibition of antimicrobial activity against Micrococcus luteus IFM 2066. The broth was applied on a column (Diaion HP-20; Mitsubishi Chemical Corp.) and washed with $50 \% \mathrm{CH}_{3} \mathrm{OH}$. Elution with $\mathrm{CH}_{3} \mathrm{OH}$ was undertaken; then it was evaporated to dryness. The residue was dissolved in distilled water, then washed with EtOAc. The active fraction was extracted with $\mathrm{BuOH}$, and the $\mathrm{BuOH}$ layer was evaporated to dryness. Then it was purified using silica gel-column chromatography $(5 \times 30 \mathrm{~cm}$; Fuji Silysia Chemical Ltd.) with a mixture of $\mathrm{CHCl}_{3} / \mathrm{CH}_{3} \mathrm{OH}$ $(10: 0$ and $7: 3)$ as eluent. The active fraction eluted by $\mathrm{CHCl}_{3} / \mathrm{CH}_{3} \mathrm{OH}(7: 3)$ was applied on preparative TLC plate (Merck and Co., Inc.) using EtOAc/ $i-\mathrm{PrOH} / \mathrm{H}_{2} \mathrm{O}(4: 2: 1$, upper phase) as a developing solution. The isolated active fraction was further purified by preparative NH-TLC plate using $\mathrm{CH}_{3} \mathrm{OH}$ as mobile phase. Finally, the active compound was applied on LH-20 column chromatography using elution solvent of $\mathrm{CHCl}_{3} / \mathrm{CH}_{3} \mathrm{OH}(1: 1)$. It gave $4.3 \mathrm{mg}$ of pure transvalencin $\mathrm{Z}$ (1) from a 2.0-liter culture.

\section{Structure Elucidation}

Physicochemical properties of transvalencin Z (1) are shown in Table 1. The IR absorbencies (FT/IR-300E; Jasco Inc.) at 1369,1615 , and $754 \mathrm{~cm}^{-1}$ indicated the presence of heterocyclic and ortho-disubstituted benzene rings in its structure (Table 2). The shoulder at $1715 \mathrm{~cm}^{-1}$ also suggested the presence of a free carboxyl group. The FABMS of 1 gave the $[\mathrm{M}+\mathrm{H}]^{+}$ion at $m / z 364$ in the positive mode and HRFAB-MS (JMS-700 and JMS-AX500 instruments; JEOL) displayed the $[\mathrm{M}+\mathrm{H}]^{+}$ion at $\mathrm{m} / \mathrm{z}$ 364.1503 (calcd. 364.1509). The molecular formula was established as $\mathrm{C}_{17} \mathrm{H}_{21} \mathrm{~N}_{3} \mathrm{O}_{6}$ based on the HRFAB-MS and NMR $\left({ }^{1} \mathrm{H},{ }^{13} \mathrm{C}\right.$, DEPT, HSQC, NOESY and HMBC) spectral data (JNM EXP-500; JEOL) (Table 3). The composition therefore indicated the presence of nine double
Table 2 Assignments of the main absorption bands $\left(\mathrm{cm}^{-1}\right)$ of the IR spectrum $(\mathrm{KBr})$ of transvalencin $\mathrm{Z}$

\begin{tabular}{ll}
\hline $3600 \sim 3100$ & $v \mathrm{NH} \& v \mathrm{OH}$ \\
2923,2865 & $v \mathrm{CH} \&$ aldehydic $v \mathrm{CH}$ \\
1653 & carboxylic $v \mathrm{C}=\mathrm{O}$ \\
1615 & $v \mathrm{C}=\mathrm{O}($ amide I) \\
1492 & aromatic $v \mathrm{C}=\mathrm{C}$ \\
1526 & $\delta \mathrm{NH}$ (amide II) \\
1369 & heterocyclic $v \mathrm{C}=\mathrm{N}$ \\
1258 & $v \mathrm{C}-\mathrm{O}$ \\
1234 & aromatic $\delta \mathrm{CH}$ \\
1078 & $v \mathrm{C}-\mathrm{N}$ \\
965 & heterocyclic $\gamma \mathrm{CH}$ \\
754 & $\gamma \mathrm{CH}$ \\
\hline
\end{tabular}

$v$ : stretching vibration, $\delta$ : deformation vibration, $\gamma$ : out-of-plane deformation vibration.

bonds or rings in the molecule. The ${ }^{1} \mathrm{H}-\mathrm{NMR}$ spectrum showed 20 hydrogen atoms, including the presence of two amide $\mathrm{NH}$ groups $(\delta 7.75$ and 7.94$)$ and a formyl proton $(\delta 7.94)$ in DMSO- $d_{6}$. The ${ }^{13} \mathrm{C}$-NMR spectrum indicated that the compound comprises 17 carbon atoms, which were assigned to five methylenes, six methines, and six quaternary carbons using DEPT and HSQC spectra.

The structure of $\mathbf{1}$ was determined as follows. A series of aromatic protons of $\delta 6.50 \sim 8.00$ in the ${ }^{1} \mathrm{H}-\mathrm{NMR}$ spectrum suggested the presence of an ortho-disubstituted benzene ring. In the COSY spectrum (Fig. 2), a spin-spin network from $\mathrm{H}-4$ to $\mathrm{H}-7$ was easily observed. The HMBC spectrum showed a cross peak between $\mathrm{H}-5$ and the carbon signal at $\delta 159.1$ (C-3) that is substituted with an oxygen-functional group and was correlated with H-7. The remaining aromatic carbon $(\mathrm{C}-2)$ was assigned to the signal at $\delta 109.1$ by the cross peaks with H-4 and H-6. The chemical shift of $\mathrm{C}-2$ suggested that the $\mathrm{C}-2$ position was substituted with a $s p^{2}$ carbon [6]. The $s p^{2}$ carbon was assigned to the signal at $\delta 165.9$ (C-1) because of long-range coupling with H-7. These data indicate the presence of the salicylate residue in this structure. The correlation between the signal at $\delta 4.48$ (an H-3 of serine residue) and 4.99 (H-2 of serine residue) was detected in the COSY spectrum. Long-range coupling between the $\mathrm{H}-3 / \mathrm{H}-2$ and $\mathrm{C}-1$ of salicylate residue (Fig. 2) indicated the presence of a 2-oxazoline ring. Eight $s p^{3}$ protons of lysine residue were connected by the COSY spectrum and the correlation between the amido proton at the $\mathrm{C}-2$ position; $\mathrm{H}-2$ of lysine residue was also detected. Long-range coupling between $\delta 2.98\left(\mathrm{H}_{2}-6\right.$ of lysine residue) and 160.7 (formic carbon) in the HMBC spectrum indicated that the $\mathrm{N}$-terminal of the side chain of lysine residue was formylated. The connection of respective 
Table $3{ }^{1} \mathrm{H}$ and ${ }^{13} \mathrm{C}$ NMR spectral data of transvalencin $\mathrm{Z}$ in DMSO- $d_{6}$

\begin{tabular}{|c|c|c|c|}
\hline Position & ${ }^{13} \mathrm{C}$ (ppm) & ${ }^{1} \mathrm{H}$ (ppm) & (mult; J, Hz) \\
\hline \multicolumn{4}{|c|}{ Salicylic acid } \\
\hline 1 & 165.9 & & \\
\hline 2 & 109.8 & & \\
\hline 3 & 159.1 & & \\
\hline 4 & 116.5 & 7.00 & (br d; 8) \\
\hline 5 & 134.0 & 7.46 & $(\mathrm{ddd} ; 1.5,7.5,8)$ \\
\hline $6^{*}$ & 119.1 & 6.94 & (br t; 7.5) \\
\hline 7 & 128.0 & 7.63 & $(\mathrm{dd} ; 1.5,7.5)$ \\
\hline \multicolumn{4}{|l|}{ Serine } \\
\hline 1 & 168.6 & & \\
\hline 2 & 67.4 & 4.99 & $(\mathrm{dd} ; 8,10)$ \\
\hline \multirow[t]{2}{*}{3} & 69.7 & 4.48 & $(\mathrm{t} ; 8)$ \\
\hline & & 4.63 & $(\mathrm{dd} ; 8,10)$ \\
\hline \multicolumn{4}{|c|}{ N-Formyl-lysine } \\
\hline 1 & 173.1 & & \\
\hline $1-\mathrm{OH}$ & & 11.76 & (br) \\
\hline $2 * *$ & 53.8 & 3.85 & (q-like; 6) \\
\hline $2-\mathrm{NH}$ & & 7.75 & $(d ; 6)$ \\
\hline \multirow[t]{2}{*}{3} & 31.7 & 1.54 & $(\mathrm{~m})$ \\
\hline & & 1.68 & (m) \\
\hline 4 & 22.5 & 1.20 & (m) \\
\hline 5 & 28.8 & 1.32 & (m) \\
\hline $6 * * *$ & 37.1 & 2.98 & (q-like; 6) \\
\hline 6-NH & & 7.94 & $(b r t ; 6)$ \\
\hline 7 & 160.7 & 7.92 & (s) \\
\hline
\end{tabular}

* (in $\mathrm{CD}_{3} \mathrm{OD}$ ) $\delta_{\mathrm{C}} 120.0, \delta_{\mathrm{H}} 6.86\left(\mathrm{dt}, J=1\right.$ and $7.5 \mathrm{~Hz}$ ). * ${ }^{*}$ The signal was changed to triplet $(\mathrm{J}=5.5 \mathrm{~Hz})$ with the addition of $\mathrm{CD}_{3} \mathrm{OD}$. ${ }^{* *}$ The signal was changed to triplet $(\mathrm{J}=6.5 \mathrm{~Hz})$ with the addition of $\mathrm{CD}_{3} \mathrm{OD}$. The assignment of the $\mathrm{OH}$ signal may be changed to $3-\mathrm{OH}$ of salicylic acid moiety ${ }^{\mathrm{D}}$.

moiety was indicated by the correlation between $\delta 7.75(2-$ $\mathrm{NH}$ of lysine residue) and 168.6 (C-1 of serine residue) in the HMBC spectrum. These data revealed that this compound comprises salicylate, a 2-oxazoline ring, and $\mathrm{N}$ formyl-lysine, suggesting a building block of mycobactintype siderophores such as mycobactins $\mathrm{J}, \mathrm{R}, \mathrm{S}$ and $\mathrm{T}$ [8]. Finally, this compound was determined to be 6-formylamino-2-\{[2-(2-hydroxy-phenyl)-4,5-dihydrooxazole-4-carbonyl]-amino\}-hexanoic acid.

Therefore, the transvalencin $\mathrm{Z}$ structure was characterized as shown in Fig. 1, except for stereochemistry at the C-2 position of serine moiety and the C-2 position of lysine moiety.

\section{Bioactive Assay}

Antimicrobial and antitumor activities of transvalencin $\mathrm{Z}$

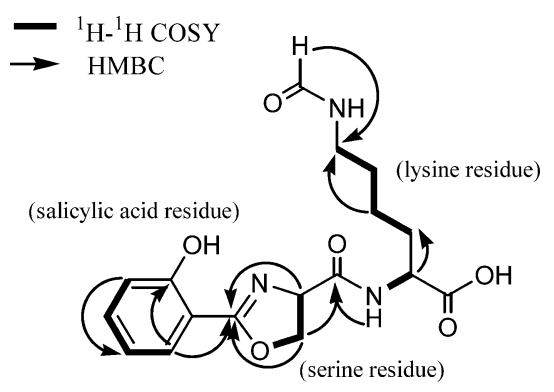

Fig. $2 \mathrm{COSY}$ and $\mathrm{HMBC}$ correlations of transvalencin Z.

Table 4 Antimicrobial activity of transvalencin Z (1) against bacteria and fungi

\begin{tabular}{lc}
\hline \multicolumn{1}{c}{ Test organisms } & MICs $(\mu \mathrm{g} / \mathrm{ml})$ \\
\hline Nocardia asteroides IFM 0319 & 1 \\
Nocardia farcinica IFM 0284 & 1 \\
Nocardia transvalensis IFM 10065 & $>64$ \\
Mycobacterium smegmatis ATCC 607 & 0.125 \\
Gordonia branchialis IFM 0150 & $<0.125$ \\
Corynebacterium xerosis IFM 2057 & 0.125 \\
Micrococcus luteus IFM 2066 & 4 \\
Bacillus subtilis PCI 189 & 16 \\
Staphylococcus aureus PCI 209P & 0.25 \\
Staphylococcus aureus CUH 3666 (MRSA) & 1 \\
Escherichia coli NIH JC-2 & $>64$ \\
Candida albicans ATCC 90028* & $>64$ \\
Aspergillus niger IFM 5368 & $>64$ \\
Cryptococcus neoformans ATCC 90112 & $>64$ \\
Tricophyton mengtagrophytes IFM 10489* & $>64$ \\
\hline
\end{tabular}

Nutrient Broth, $37^{\circ} \mathrm{C}, 24$ hours, ${ }^{*}: 37^{\circ} \mathrm{C}, 72$ hours. MRSA: Methicillinresistant $S$. aureus.

(1) were tested; the results are shown in Table 4. Antibiotic 1 was active against Gram-positive bacteria, especially against acid-fast bacteria such as Corynebacterium xerosis, Gordonia branchialis and Mycobacterium smegmatis, and their MIC values were less than $0.125 \mu \mathrm{g} / \mathrm{ml}$. The antibiotic was moderately active against Gram-positive bacteria such as Micrococcus luteus $(4.0 \mu \mathrm{g} / \mathrm{ml})$ and Staphylococcus aureus $(0.25 \mu \mathrm{g} / \mathrm{ml})$, but not active against Gram-negative bacteria, fungi and tumor cells. A similar compound, methyl-2-(2'-hydroxyphenyl)-2-oxazoline-4-carboxylate (salicylic acid and serine moieties of $\mathbf{1}$ ), was isolated from Actinomadura species [9]. Antimicrobial activity of $\mathbf{1}$ against Gram-positive bacteria was stronger than that of the simple compound, but its cytotoxic activity was weaker than that of this compound.

Compound 1 has three binding sites of the six found on 
nocobactin-type and mycobactin-type siderophores [10]. For that reason, it is expected that $\mathbf{1}$ complexes with an iron ion $(1: \mathrm{Fe}=2: 1$ complex $)$. The present study confirmed that 1 has no siderophore activity because its antimicrobial activity is not abolished by addition of $\mathrm{Fe}^{+3}$ ion into the medium. Furthermore, the color of the medium was not changed by the addition of the $\mathrm{Fe}^{+3}$ ion (Fe-nocobactin and Fe-mycobactin complexes are red) [11]. The inference is also supported by the fact that $\mathbf{1}$ shows no cytotoxic activity.

Most Nocardia strains isolated from clinical specimens were found to produce bioactive secondary metabolites with salicylic acid just as siderophores. For that reason, we were interested in the biosynthesis gene of salicylic acid in Nocardia clinical isolates. Our preliminary PCR amplification studies using salicylic acid biosynthesis genes [12] indicated that most Nocardia species possess gene(s) for production of salicylic acid containing compounds. Although most such compounds are considered to belong to salicylic acid containing siderophores, there remains the strong possibility of finding novel bioactive secondary metabolites containing salicylic acid, similar to the transvalencin $\mathrm{Z}$ antibiotic studied here.

\section{References}

1. Beaman BL, Beaman L. Nocardia species. Host-parasite relationships. Clin Microbiol Rev 7: 213-264 (1994)

2. Kageyama A, Yazawa K, Ishikawa J, Hotta K, Nishimura K, Mikami Y. Nocardial infections in Japan from 1992 to 2001, including the first report of infection by Nocardia transvalensis. Eur J Epidemiol 19: 383-389 (2004)

3. Nemoto A, Hoshino Y, Yazawa K, Ando A, Mikami Y, Komaki H, Tanaka Y, Grafe U. Asterobactin, a new siderophore group antibiotic from Nocardia asteroides. J
Antibiot 55: 593-597 (2002)

4. Tsuda M, Yamakawa M, Oka S, Tanaka Y, Hoshino Y, Mikami Y, Sato A, Fujiwara H, Ohizumi Y, Kobayashi J. Brasilibactin A, a cytotoxic compound from actinomycete Nocardia brasiliensis. J Nat Prod 68: 462-464 (2005)

5. Harada K, Tomita K, Fujii K, Masuda K, Mikami Y, Yazawa $\mathrm{K}$, Komaki H. Isolation and structural characterization of siderophores, madurastatins, produced by a pathogenic Actinomadura madurae. J Antibiot 57: 125-135 (2004)

6. Hoshino Y, Mukai A, Yazawa K, Uno J, Ando A, Mikami Y, Fukai T, Ishikawa J, Yamaguchi K. Transvalencin A, a thiazolidine zinc complex antibiotic produced by a clinical isolate of Nocardia transvalensis. II. Structure elucidation. J Antibiot 57: 803-807 (2004)

7. Hoshino Y, Mukai A, Yazawa K, Uno J, Ishikawa J, Ando A, Fukai T, Mikami Y. Transvalencin A, a thiazolidine zinc complex antibiotic produced by a clinical isolate of Nocardia transvalensis. I. Taxonomy, fermentation, isolation and biological activities. J Antibiot 57: 797-802 (2004)

8. Ishikawa J, Yamashita A, Mikami Y, Hoshino Y, Kurita H, Hotta K, Shiba T, Hattori M. The complete genomic sequence of Nocardia farcinica IFM 10152. Proc Nat Acad Sci USA 101: 14925-14930 (2004)

9. Sasaki T, Otani T, Yoshida K, Unemi N, Hamada M, Takeuchi T. Discovery of methyl-2-(2'-hydroxyphenyl)-2oxazoline-4-carboxylate as a secondary metabolite from Actinomadura sp. J Antibiot 50: 881-883 (1997)

10. Hough E, Rogers D. The crystal structure of ferrimycobactin $\mathrm{P}$, a growth factor for the mycobacteria. Biochem Biophys Res Commun 57: 73-77 (1974)

11. Ratledge C, Patel PV. Lipid-soluble, iron-binding compounds in Nocardia and related organisms. In The biology of the nocardiae. Ed., Goodfellow M, et al., pp. 372-385, Academic Press, London (1976)

12. Vergne AF, Walz AJ, Miller MJ. Iron chelators from mycobacteria (1954-1999) and potential therapeutic applications. Nat Prod Rep 17: 99-116 (2000) 